\title{
The NEID precision radial velocity spectrometer: port adapter overview, requirements, and test plan
}

Sarah E. Logsdon, Michael W. McElwain, Qian Gong, Ming Liang, Fernando Santoro, et al.

Sarah E. Logsdon, Michael W. McElwain, Qian Gong, Ming Liang, Fernando Santoro, Christian Schwab, Chad Bender, Cullen Blake, Samuel Halverson, Fred Hearty, Emily Hunting, Kurt P. Jaehnig, Suvrath Mahadevan, Andrew J. Monson, Jeffrey W. Percival, Jayadev Rajagopal, Lawrence Ramsey, Arpita Roy, Michael P. Smith, Ryan C. Terrien, Erik Timmermann, Phil Willems, Marsha J. Wolf, Jason Wright, "The NEID precision radial velocity spectrometer: port adapter overview, requirements, and test plan

," Proc. SPIE 10702, Ground-based and Airborne Instrumentation for Astronomy VII, 1070267 (27 July 2018); doi: 10.1117/12.2312209

Event: SPIE Astronomical Telescopes + Instrumentation, 2018, Austin, Texas, United States 


\title{
The NEID precision radial velocity spectrometer: Port Adapter overview, requirements, and test plan
}

Sarah E. Logsdon ${ }^{a, o}$, Michael W. McElwain ${ }^{a}$, Qian Gong ${ }^{a}$, Ming Liang $^{b}$, Fernando Santoro $^{c}$, Christian Schwab ${ }^{d, e}$, Chad Bender ${ }^{f}$, Cullen Blake ${ }^{g}$, Samuel Halverson ${ }^{h, p}$, Fred Hearty ${ }^{i, j}$, Emily Hunting $^{b}$, Kurt P. Jaehnig ${ }^{k}$, Suvrath Mahadevan ${ }^{i, j}$, Andrew J. Monson ${ }^{i, j}$, Jeffrey W. Percival ${ }^{k}$, Jayadev Rajagopal ${ }^{b}$, Lawrence Ramsey ${ }^{i, j}$, Arpita Roy ${ }^{l}$, Michael P. Smith ${ }^{k}$, Ryan C. Terrien ${ }^{m}$, Erik Timmerman ${ }^{b}$, Phil Willems ${ }^{n}$, Marsha J. Wolf ${ }^{k}$, Jason Wright ${ }^{i, j}$

${ }^{a}$ NASA Goddard Space Flight Center, 8800 Greenbelt Rd, Greenbelt, MD, 20771, USA;

${ }^{b}$ National Optical Astronomy Observatory, 950 N Cherry Ave, Tucson, AZ, 85719, USA;

${ }^{c}$ ASTRO LLC, PO Box 623, Los Lunas, NM 87031, USA;

${ }^{d}$ Centre for Astronomy, Astrophysics \& Astrophotonics, Macquarie University, NSW 2109, Australia;

e Australian Astronomical Observatory, PO Box 915, NSW 2109, Australia;

${ }^{f}$ Steward Observatory, University of Arizona, Tucson, AZ 85721;

${ }^{g}$ Department of Physics \& Astronomy, University of Pennsylvania, 209 South 33rd Street, Philadelphia, PA, USA, 19104;

${ }^{h}$ Kavli Institute for Astrophysics and Space Research, Massachusetts Institute of Technology,

77 Massachusetts Avenue, 37-241, Cambridge, MA 02139;

${ }^{i}$ Department of Astronomy \& Astrophysics, The Pennsylvania State University, 525 Davey

Laboratory, University Park, USA, 16802;

${ }^{j}$ Center for Exoplanets \& Habitable Worlds, The Pennsylvania State University, 525 Davey

Laboratory, University Park, USA, 16802;

${ }^{k}$ Washburn Astronomical Laboratories, University of Wisconsin-Madison, 475 N Charter St., Madison, WI, 53706, USA;

${ }^{l}$ California Institute of Technology, Pasadena, CA, 91125, USA;

${ }^{m}$ Carleton College, Northfield, MN, 55057, USA;

$n$ Jet Propulsion Laboratory, Pasadena, CA 91109, USA;

o NASA Postdoctoral Program Fellow;

$p$ Sagan Fellow

\begin{abstract}
The NEID spectrometer is an optical (380-930 nm), fiber-fed, precision Doppler spectrometer currently in development for the WIYN $3.5 \mathrm{~m}$ telescope at Kitt Peak National Observatory as part of the NN-EXPLORE partnership. Designed to achieve a radial velocity precision of $<30 \mathrm{~cm} / \mathrm{s}$, NEID will be sensitive enough to detect terrestrial-mass exoplanets around low-mass stars. Light from the target stars is focused by the telescope to a bent Cassegrain port at the edge of the primary mirror mechanical support. The specialized NEID "Port Adapter" system is mounted at this bent Cassegrain port and is responsible for delivering the incident light from the telescope to the NEID fibers. In order to provide stable, high-quality images to the science instrument, the Port Adapter houses several sub-components designed to acquire the target stars, correct for atmospheric dispersion, stabilize the light onto the science fibers, and calibrate the spectrometer by injecting known wavelength sources such as a laser frequency comb. Here we provide an overview of the overall opto-mechanical design and
\end{abstract}

Further author information: (Send correspondence to Sarah E. Logsdon)

Sarah E. Logsdon.: E-mail: sarah.e.logsdon@nasa.gov, Telephone: 1301 614-6733

Ground-based and Airborne Instrumentation for Astronomy VII, edited by Christopher J. Evans, Luc Simard, Hideki Takami, Proc. of SPIE Vol. 10702, 1070267 - @ 2018 SPIE · CCC code: 0277-786X/18/\$18 · doi: 10.1117/12.2312209 
system requirements of the Port Adapter. We also describe the development of system error budgets and test plans to meet those requirements.

Keywords: radial velocity instrumentation; exoplanets; systems engineering

\section{INTRODUCTION}

The NEID* spectrometer (PI Suvrath Mahadevan) is an extreme precision Doppler spectrometer funded by NASA through the NASA-NSF EXoPLanet Observational REsearch (NN-EXPLORE) partnership. Expected to be on sky in 2019, NEID will be a facility class instrument on the WIYN $3.5 \mathrm{~m}$ telescope on Kitt Peak in Arizona and will be available to the larger astronomical community through the National Optical Astronomy Observatory (NOAO) telescope allocation committee (TAC) process ${ }^{\dagger}$. Designed to achieve an instrumental measurement precision of $<30 \mathrm{~cm} / \mathrm{s}$, NEID will provide critical follow-up for exoplanet candidates discovered using transit photometery missions like Kepler ${ }^{1},{ }^{2} K_{2},{ }^{3}$ and the recently launched $T E S S^{4}$ mission. Such missions have been successful at revealing exoplanet candidates and setting constraints on their radii, but radial velocity measurements are required for confirming these candidates and measuring dynamical masses, which sets constraints on planetary densities when combined with the transit-based radii.

In order to achieve the expected radial velocity precision, the NEID spectrometer is isolated from the telescope in a pressure- and temperature-controlled environment. Thus, the NEID system also includes a "Port Adapter" which attaches directly to a bent Cassegrain port on the WIYN telescope and couples the light from the telescope to the science fibers. Briefly, NEID covers the 380-930 nm wavelength region and has two modes: high-resolution (HR) and high-efficiency (HE). The HR mode is intended for bright targets $(\mathrm{V}<12 \mathrm{mag})$ and has a resolution of $\sim 100,000$. The HE mode is intended for faint stars and sub-optimal observing conditions and has a resolution of $\sim 60,000$. This proceedings provides an overview of the Port Adapter subsystem. The reader is referred to Bender et al. (this conference) $)^{5}$ for an overview of the spectrometer, including key design features and current status as well as brief description of the NEID calibration scheme. The optical design of the spectrometer has already been described in Schwab et al. (2016). ${ }^{6}$ A description of the Port Adapter optical design, including a discussion of design trade-offs, can be found in Schwab et al. (this conference). ${ }^{7}$

As outlined in the abstract, the Port Adapter system is responsible for conducting several critical actions in the NEID system, including:

- Acquiring target stars

- Guiding using fast, tip/tilt wavefront correction (see Percival et al. this conference ${ }^{8}$ for details)

- Atmospheric dispersion correction

- Stabilizing target light onto the science fibers

- Calibrating the spectrometer by injecting known wavelength sources

In the following sections, we describe the opto-mechanical design of the Port Adapter (Section 2), summarize the key system requirements for the NEID Port Adapter (Section 3), describe the use of detailed error budgets to track the expected performance of the instrument (Section 3), and describe the development of in-lab and on-sky test plans to verify performance (Section 4). We end this proceedings with a brief description of the Port Adapter's current status.

\footnotetext{
${ }^{*}$ NEID is both an acronym and a word. NEID stands for the NN-Explore Exoplanet Investigations with Doppler Spectroscopy and also derives from the O'odham word 'neid' meaning "to see/visualize." Kitt Peak Observatory is located on the Tohono O'odham Reservation.

${ }^{\dagger} \mathrm{NOAO}$ time constitutes $40 \%$ of the available nights on WIYN.
} 


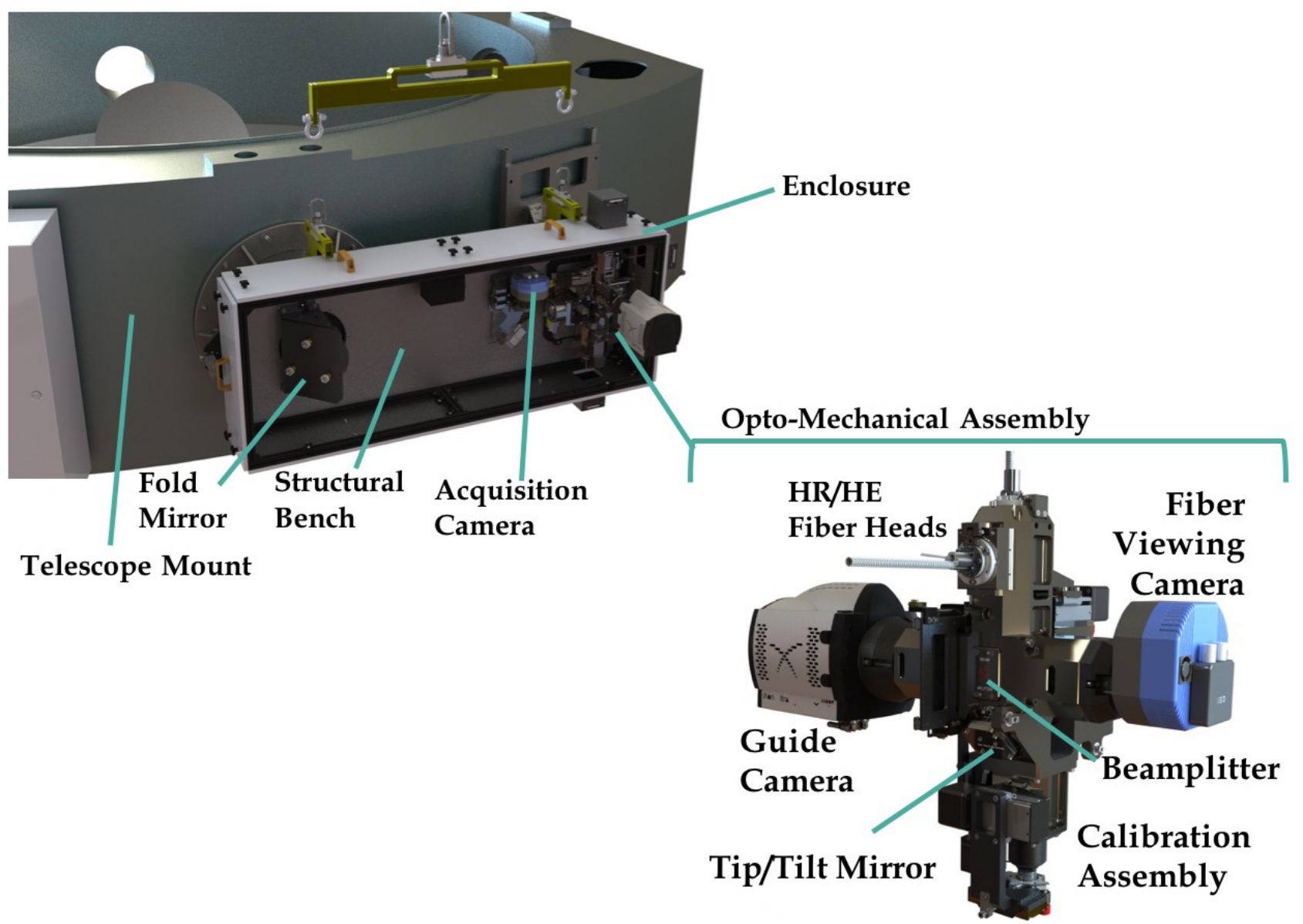

Figure 1. 3D rendering of the main NEID Port Adapter module mounted to a bent Cassegrain port on the WIYN telescope. The lifting fixture for Port Adapter installation is also shown. Inset: Zoom in on the opto-mechanical assembly, a modular unit containing the main subsystems of the Port Adapter.

\section{OPTO-MECHANICAL DESIGN}

The Port Adapter is designed to provide a stiff mechanical support which allows alignment of its optical components to be highly stable and repeatable over short and long observation timescales, at all telescope orientations and operational temperatures $\left(-5\right.$ to $\left.25^{\circ} \mathrm{C}\right)$.

The Port Adapter mechanical design is characterized by its modularity, with the following major modules: structural bench, enclosure, fold mirror, acquisition camera, and opto-mechanical assembly (see Figure 1). The opto-mechanical assembly includes several sub-assemblies that are designed to be both modular and in close proximity to each other, which allows significant reduction of built-up tolerances between individual sub-modules. Following the order that they appear to the incoming beam of light these sub-modules are: collimator triplet, atmospheric dispersion corrector (ADC), calibration fiber injection unit, tip-tilt mirror, beamsplitter, HR/HE fiber head assembly, guide camera assembly, and fiber viewing camera assembly. Light transmitted by the beamsplitter is incident on the HR/HE fiber head assembly. The light reflected by the beamsplitter passes to the guide camera assembly, and light that is reflected off of the fibers is reflected off of the back side of the beamsplitter into the fiber viewing camera assembly (see Figure 2 and Schwab et al. this conference ${ }^{7}$ for details on the optical design). The modularity approach has facilitated the design process, procurement and fabrication, and will be key during pre-alignment and performance testing in the lab, as well as installation and alignment at the telescope site. 


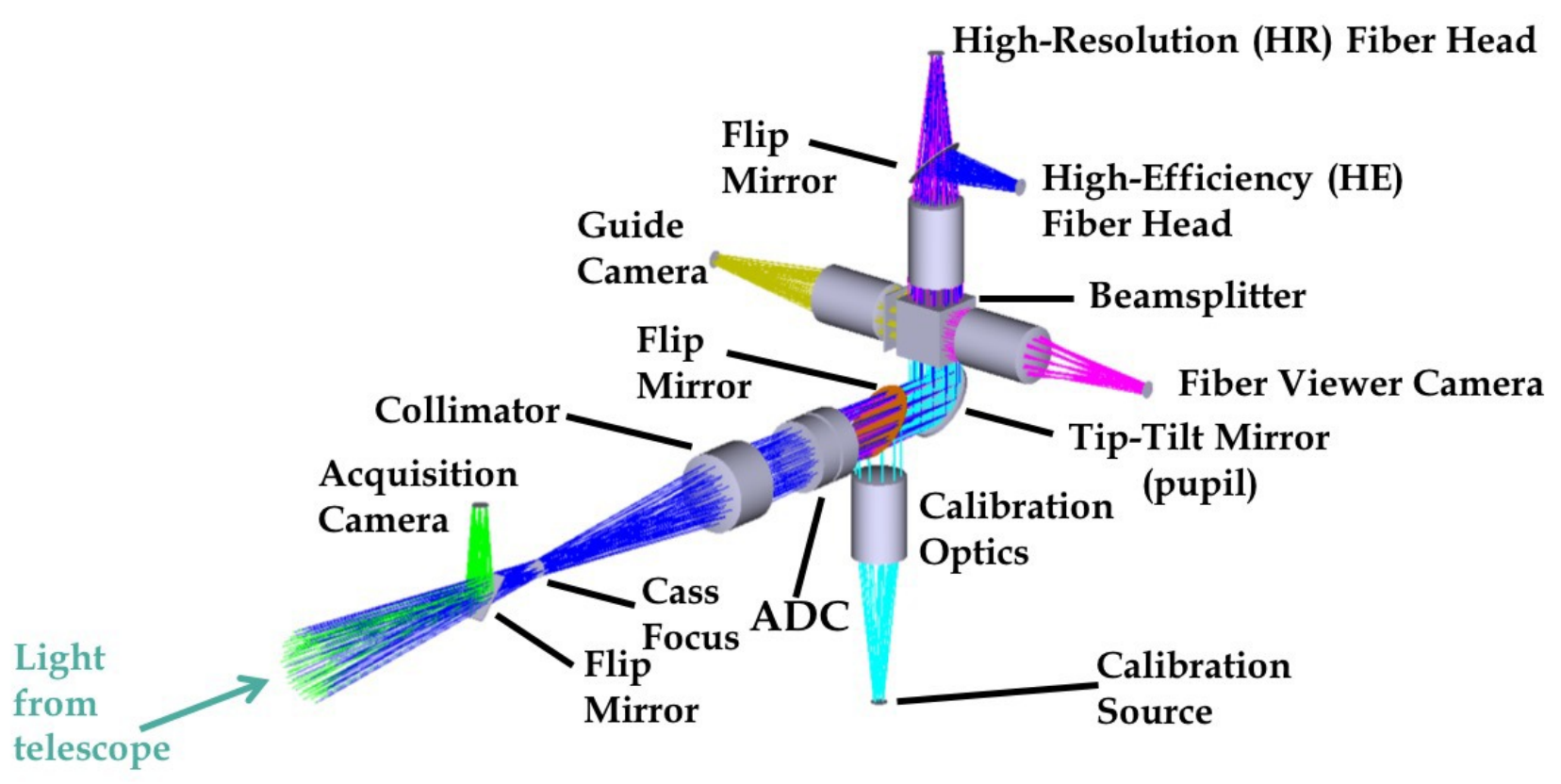

Figure 2. A ray trace diagram of the main Port Adapter optics. The incident light from the WIYN telescope's tertiary mirror reflects off of a fold mirror (not shown) and into the optics shown. Note: This figure and a more detailed discussion of the key Port Adapter optics can also be found in Schwab et al. (this conference). ${ }^{7}$

The substantial opto-mechanical housing is made of six Invar-36 blocks and is designed to precisely and stably support all opto-mechanical sub-modules. The Invar parts are electroless nickel plated with internal surfaces blackened using Aeroglaze Z306. The opto-mechanical assembly is kinematically mounted on the structural bench via Spherolinders. ${ }^{9}$ The stiffness of the housing is optimized via finite element analysis (FEA) so as to minimize differential flexure between internal optical elements during or between observations.

The structural bench interfaces rigidly with one of the WIYN telescope's bent Cassegrain ports, and all the other modules within the main Port Adapter system. The structural bench is also supported to the telescope mount using a bipod-flexure near its Airy point for lower flexure and improved mass distribution (with a first resonant frequency of $119 \mathrm{~Hz}$ ). The bipod-flexure is made compliant along the instrument optical axis to minimize creeping between the various mechanical subsystems and to maintain optical alignment stability over short and long term observation time. The structural bench and bipod-flexure are made of steel A-36 to match the material of the telescope mount and thus provide an athermal design. The bipod-flexure can be adjusted (shimmed) during first assembly to place its upper mounting surface at the correct location with minimal stress. Once installed, the bipod-flexure does not need to be removed.

The Port Adapter will be nearly symmetric to a horizontal plane at the bent Cassegrain port (with lower imbalance). A light-weight enclosure is designed around the Port Adapter to provide stray light management, a cleaner optical environment, and access to all opto-mechanics. The structural bench assembly also includes an environmental cover to block light from/to the telescope during calibrations. The cover will be closed when the instrument is not in use to protect it from humidity and dust.

Adjacent to the main Port Adapter system is the Coherent Fiber Bundle (CFB) subsystem (see Figure 3). As discussed in more detail in Section 3, the purpose of the CFB assembly is to register the HR and HE fiber heads to the Guide Camera, to enable precise stellar image positioning on the science fiber. There are three CFBs and three sky/spare science fibers surrounding the central science fiber on both the HR and HE fiber heads (see Figure 4). For both fiber heads, the CFBs are equally spaced $120^{\circ}$ from each other and are all located at a radius of $1 \mathrm{~mm}\left(\sim 14.7^{\prime \prime}\right)$. The sky fibers are also equally spaced $120^{\circ}$ from each other and are located at a radius of $1.5 \mathrm{~mm}\left(\sim 22^{\prime \prime}\right)$ (see Figure 4$)$. The sky fibers are clocked $60^{\circ}$ from the CFBs. The science and sky 


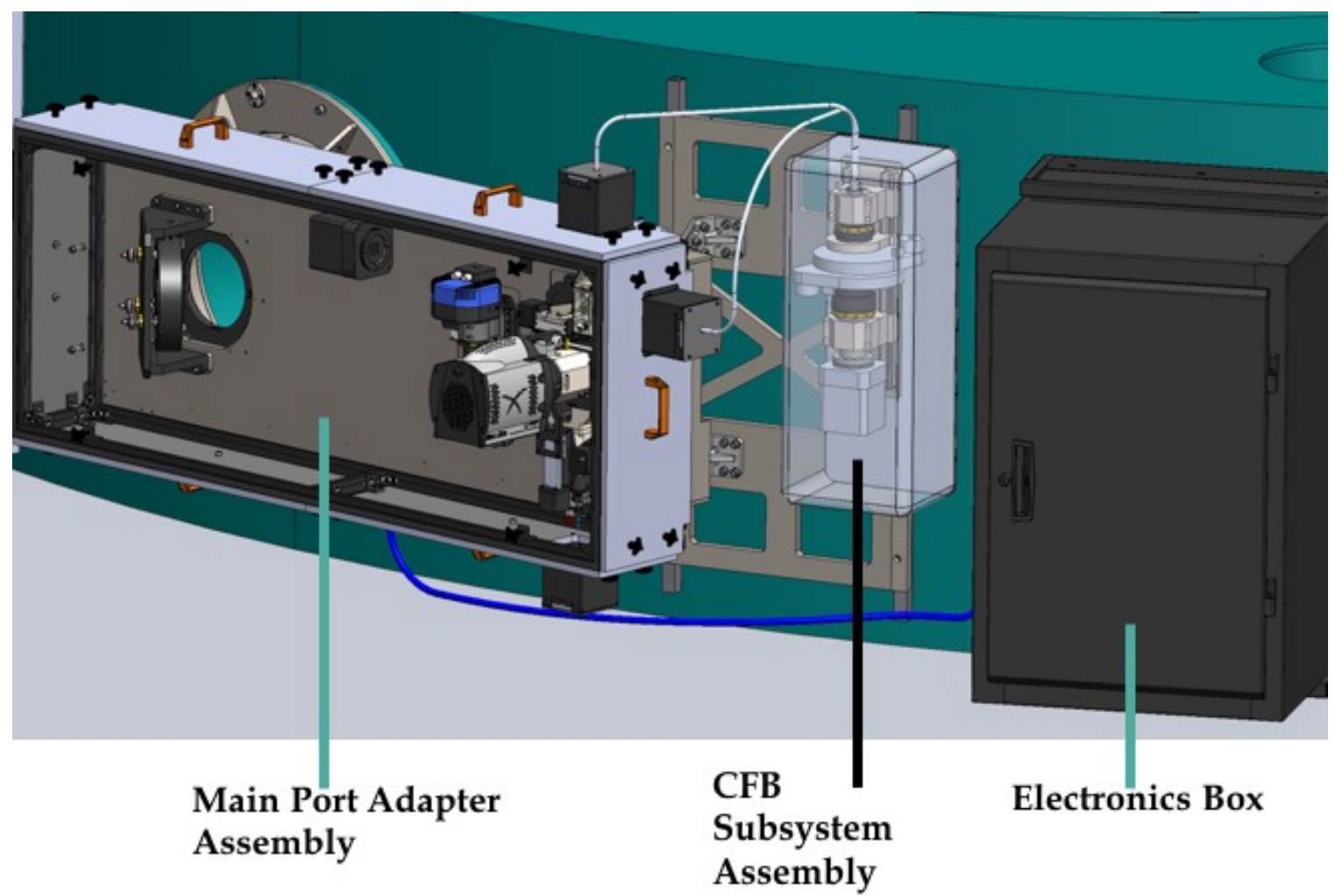

Figure 3. Rendering of the entire Port Adapter assembly including the Coherent Fiber Bundle (CFB) subsystem, which is mounted adjacent to the main Port Adapter assembly. The CFB assembly images six CFBs simultaneously (three each from the HR and HE fiber heads) and will be used to triangulate the location of the science target on the fiber head to enable accurate placement of the science target on the science fiber.

fibers from each fiber head are routed to the spectrometer, but the CFBs are routed to the CFB assembly. The ends of the three CFBs from each fiber head (HR/HE) are all mounted in a hemisphere connector. The two connectors are identical except for a unique locating pin on each. The CFBs are all equally spaced. The two hemisphere connectors are then joined together into one circular plate that interfaces with the CFB assembly. Once interfaced with the assembly, the light from the CFBs passes through a collimator, two COTS filter wheels from Finger Lakes Instrumentation (one each for neutral density filters and broadband filters) and a camera before being simultaneously imaged onto a detector. The CFB optics are optimized over the 500-600 nm range.

\section{SYSTEM REQUIREMENTS AND ERROR BUDGETS}

The overall NEID design is driven by a comprehensive instrumental radial velocity (RV) error budget, the details of which can be found in Halverson et al. (2016). ${ }^{10}$ This radial velocity error budget sets strict requirements on many aspects of the NEID design, including the Port Adapter. The Port Adapter requirements are primarily motivated by the need for stable fiber injection. In practice, this need for stability sets requirements on three fundamental Port Adapter parameters: 1) guiding/stellar image positioning precision, 2) atmospheric dispersion correction, and 3) chief ray angle deviation. As discussed in Halverson et al. (2016), ${ }^{10}$ both guiding errors and uncorrected atmospheric dispersion contribute to near-field scrambling error in the fibers. Guiding precision also impacts the far-field scrambling error, as does a change in the incident chief ray angle. In order to quantify the design and measurement errors that will impact our ability to meet these system requirements, we have adopted an error budget accounting of the key Port Adapter requirements following a traditional systems engineering approach (e.g., Lightsey et al. 2014 ${ }^{11}$ ). Below we outline the specific requirements for atmospheric dispersion correction, chief ray angle deviation, and stellar image positioning precision, which takes stable guiding from the 


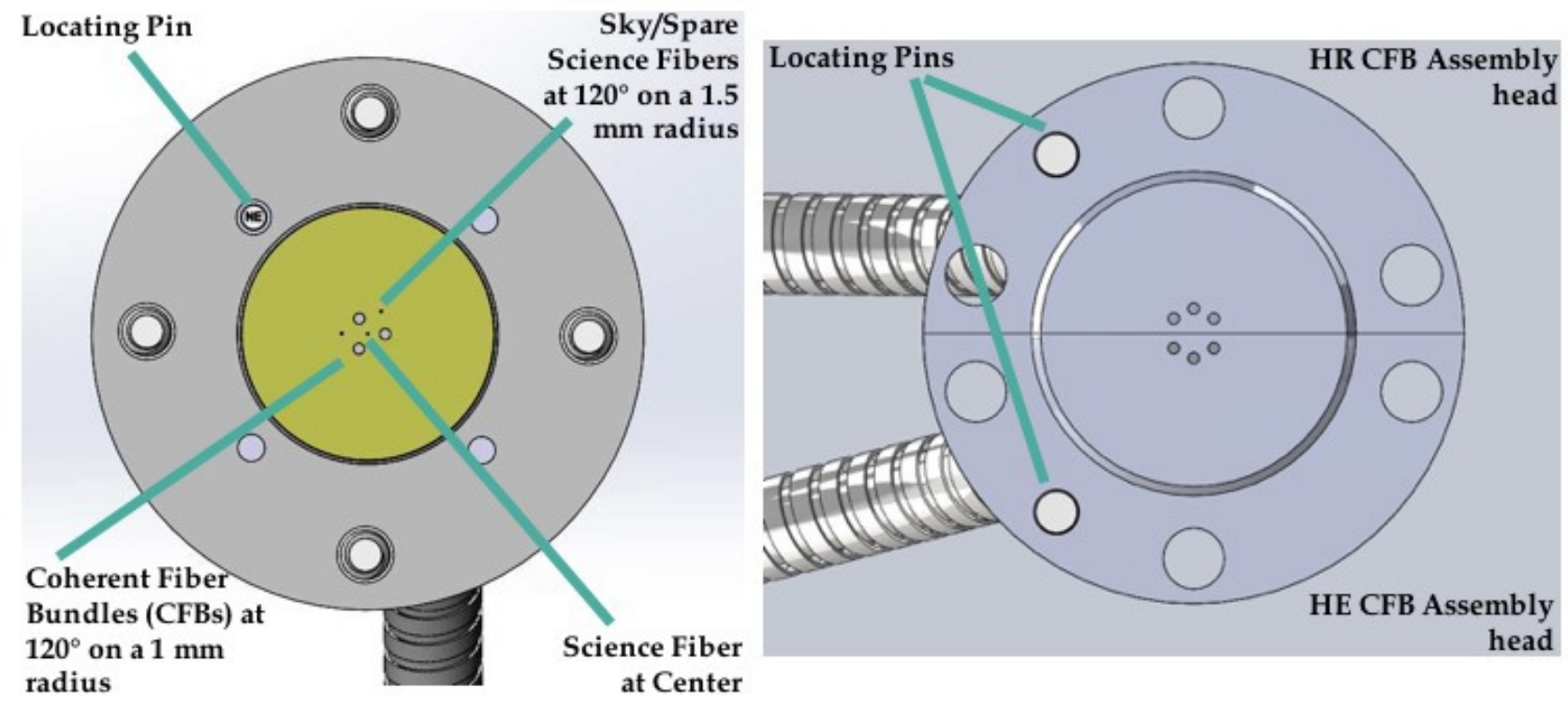

Figure 4. Renderings of an HR/HE NEID fiber head (left) and the combined HR/HE CFB Assembly head (right). Both the HR and HE fiber heads feature a central science fiber $(62.5 \mu$ m diameter for HR; $102 \mu$ m diameter for HE) surrounded by three coherent fiber bundles (CFBs; $380 \mu \mathrm{m}$ quality area diameter) at a radius of $1 \mathrm{~mm}$ and three sky/spare science fibers at a radius of $1.5 \mathrm{~mm}$. The CFB Assembly head is made up of two hemispheres that are identical with the exception of unique locating pins and are mounted together to form a circular connector. The three CFBs from each NEID fiber head are routed to one hemisphere and all six CFBs are simultaneously imaged on the CFB imager.

WIYN telescope and the Port Adapter's fast, tip/tilt correction (see Percival et al. this conference ${ }^{8}$ ) as input. As an example of our methodology, we also describe our working error budget for the stellar image positioning requirement.

The atmospheric dispersion requirement for the Port Adapter states that the peak-to-valley displacement error across the entire NEID bandpass $(380-930 \mathrm{~nm})$ shall not exceed $0.1^{\prime \prime}$ for zenith angles from $0-58^{\circ}$, and shall not exceed $0.2^{\prime \prime}$ between $58-71^{\circ}$. The Port Adapter atmospheric dispersion corrector (ADC) is made up of a pair of Amici prism doublets which have been designed to meet our requirement. A detailed discussion of the design trade-offs considered in order to meet the atmospheric dispersion requirement can be found in Schwab et al. (this conference). ${ }^{7}$ On-sky ADC performance is dependent on the atmospheric model chosen, which is in turn dependent on input temperature and pressure information. To verify on-sky performance of the ADC, we are also exploring a diffractive technique pioneered for ground-based adaptive optics coronagraphy to measure residual atmospheric dispersion over the entire NEID bandpass (see Pathak et al. 2016 ${ }^{12}$ ).

The chief ray requirement specifies that the chief ray must be within $3^{\prime}$ of the nominal optical axis of the science fiber for the central $5^{\prime \prime} \mathrm{FOV}$ at the science fiber bundles. In practice, the chief ray angle requirement comprises both a static component and a stability component. The static component considers the known alignment precision of the WIYN telescope and motivates the alignment precision of the Port Adapter optics themselves. The stability component includes the flexure (gravitational and thermal) of the telescope and Port Adapter system which also sets constraints on the implementation of the ADC prisms, as rotating the ADC prisms can change the chief ray angle incident on the science fibers (again see Schwab et al. this conference ${ }^{7}$ for details).

The stellar image positioning requirement for bright stars $(\mathrm{V}<12 \mathrm{mag})$ states that the centroid of the stellar PSF must be maintained within the central $0.05^{\prime \prime}$ (3.4 microns for a $\mathrm{f} / 4$ beam) of the science fiber under median seeing and wind conditions. This requirement relaxes to $0.2^{\prime \prime}$ for faint stars (12 mag $\left.<\mathrm{V}<16 \mathrm{mag}\right)$. The error budget for the stellar image positioning requirement is shown in Figure 5 and detailed below. To achieve a $0.05^{\prime \prime}$ $\left(0.2^{\prime \prime}\right)$ positioning precision on the science fiber requires both accurate guiding (again see Percival et al. this 
conference $^{8}$ for a discussion of the Port Adapter's fast, tip/tilt correction) and an accurate registration between the guide camera and the HR (HE) fiber head. As alluded to in Section 2, the goal of the CFB assembly is to facilitate that registration. By imaging the target star sequentially in each of the three CFBs while guiding, we will be able to triangulate the star's location on the fiber head in relation to the guide camera. Combining this registration of the three CFBs to the guide camera with lab measurements of the fiber head metrology will determine the position of the science fiber in relation to the three CFBs on the head, enabling us to accurately place the target star on the science fiber. The use of CFBs for image positioning builds on legacy from the Habitable Planet Finder (HPF; PI-Suvrath Mahadevan). The HPF (see e.g. Mahadevan et al. 2012, 2014 ${ }^{13,14}$ ) is a near-infrared precision Doppler spectrometer at the Hobby-Eberly Telescope at McDonald Observatory (delivered in 2017). The HPF also employs CFBs (in this case, two) as part of its target acquisition scheme. CFBs are more-ideally suited for locating a target star than a single science fiber as CFBs have both more pixels (fibers) and a larger field-of-view than single science fibers. For example, the NEID CFBs each have $\sim 4 \mathrm{k}$ pixels and a quality image diameter of $0.38 \mathrm{~mm}\left(\sim 5.6^{\prime \prime}\right)$, compared to the HR and HE science/sky fibers, which have diameters of $62.5 \mu \mathrm{m}\left(\sim 0.92^{\prime \prime}\right)$ and $102 \mu \mathrm{m}\left(\sim 1.5^{\prime \prime}\right)$ respectively.

Our stellar image positioning error budget takes a top-down, bottom-up approach: we start with the positioning requirement and then identify instrumental/environmental sources of error that impact that requirement. Once the sources of error are identified, we work backwards, measuring (where possible) or estimating the contribution of each error source to the budget and tabulating all of the errors together to predict our on-sky precision. Error budgets are critical in every stage of the Port Adapter development. They have helped inform design decisions and have motivated in-lab and on-sky test planning. As more measured results become available from lab testing and ultimately from on-sky commissioning, we update the error budgets accordingly and reallocate errors as needed. The error budget for the stellar image positioning requirement (Figure 5) includes both static and stability (dynamic, i.e. flexure) error components. As discussed above, accurately placing the target star on the science fiber is dependent on both accurate guiding and an accurate mapping between the guide camera and the fiber head. The first level of our error budget incorporates both of those dependencies: guide camera residual error and the error in our ultimate guide camera to fiber head map, as well as an error term for any residual flexure in the main Port Adapter assembly. Because there is a separate requirement on the tip/tilt correction, the only guiding error we include here is residual error in our centroiding precision, which we conservatively hold as 1/10th of a pixel (1.3 $\mu \mathrm{m}$ for $13 \mu \mathrm{m}$ pixels). Residual errors in the guide camera to fiber head map include contributions from the in-lab metrology, which we expect to be accurate at the $\sim 1 \mu \mathrm{m}$ level based on similar measurements for HPF, and the x-y positional information derived from the three CFBs, which we label "Science Fiber Centroiding" in the error budget. We conservatively estimate the error from the CFB assembly as the error from three single, independent CFB measurements added in quadrature. For each individual CFB measurement we include three error terms: 1) a CFB Assembly flexure term (labeled "CFB Head to Detector Stability"), 2) an individual fiber bundle rotation term (note: this term can be mitigated by placing the star at the center of each fiber bundle and likely only impacts early commissioning work), and 3) a centroiding precision term (labeled "CFB Tail to Detector Residual"), which, as with the guide camera, we conservatively estimate to be 1/10th of a pixel. Starting from the bottom of the budget, the terms in each row are added in quadrature and fed up to the next level. Based on our current prediction we are within our stellar image positioning requirement.

\section{TEST PLANS AND CURRENT STATUS}

As discussed above, our in-lab and on-sky test planning stems from our error budgets. For each line item in an error budget, we identify a verification test and, where possible, a secondary cross check. In most cases, we plan to perform the verification test in the lab and perform an on-sky cross check during commissioning. For example, the CFB Assembly flexure error will be estimated in the lab at the University of Wisconsin-Madison (UW) using an LED ring installed in front of the fiber heads to illuminate the CFBs, which enables us to measure the CFB centroids (using a centroiding algorithm that has already been tested on a HPF CFB at UW), and a rotary table that tilts from $0-90^{\circ}$ to generate gravitational flexure. This test will be repeated on-sky during commissioning.

The Port Adapter is currently in the final procurement phase. The major mechanical and optical components are either currently in fabrication or have been completed. Software development is progressing along with the opto-mechanical system. Optical alignment, system integration, and testing will begin this summer (2018) in the 


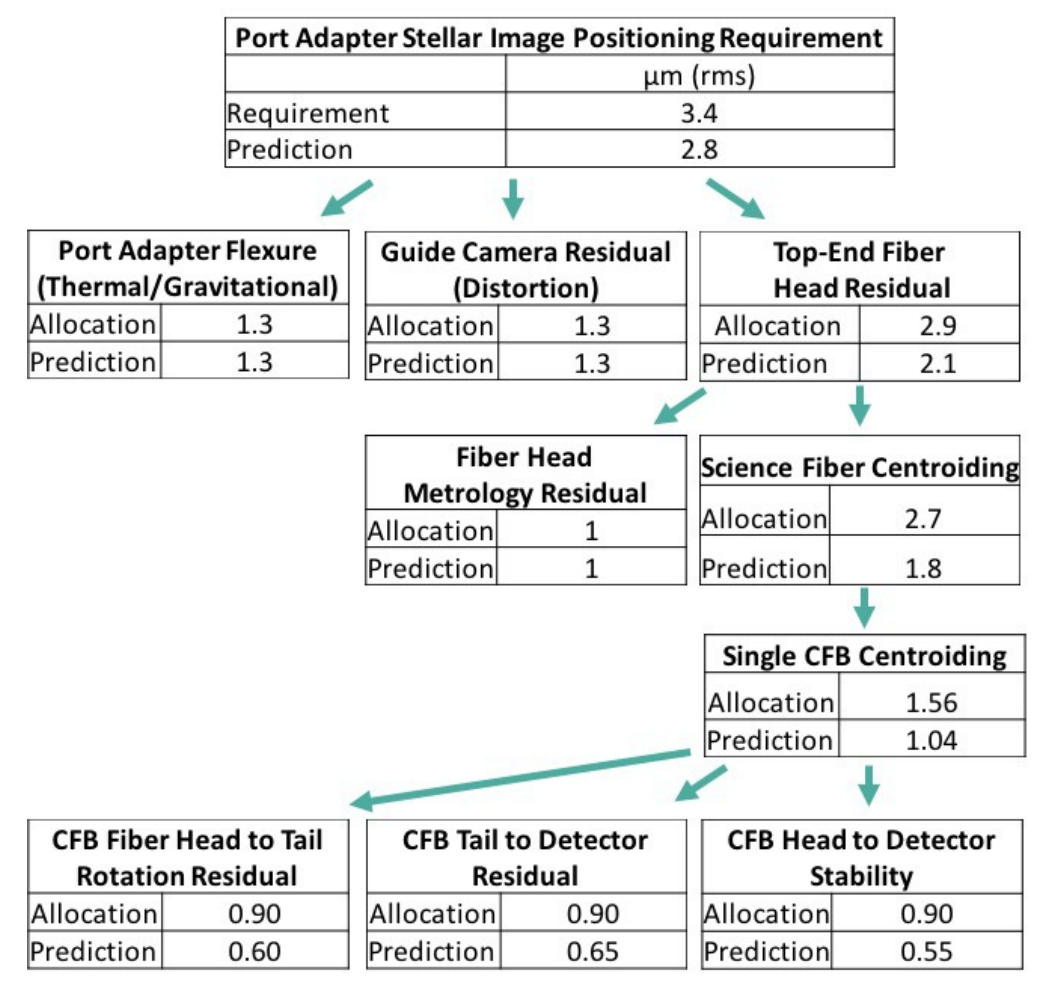

Figure 5. Error budget for NEID's stellar image positioning requirement. All values are reported in units of $\mu \mathrm{m}$ (rms). This error budget includes both static and dynamic terms, for both the guide camera (main port assembly) and the CFB assembly.

Washburn Astronomical Laboratories at the University of Wisconsin-Madison, with delivery of the Port Adapter to NOAO expected in late-2018B.

\section{ACKNOWLEDGMENTS}

Sarah Logsdon is supported by an appointment to the NASA Postdoctoral Program at NASA Goddard Space Flight Center, administered by USRA through a contract with NASA. This work was performed, in part, by Samuel Halverson and Christian Schwab under contract with the Jet Propulsion Laboratory (JPL) funded by NASA through the Sagan Fellowship Program executed by the NASA Exoplanet Science Institute. Christian Schwab also acknowledges the support of an ARC DECRA fellowship. The Center for Exoplanets and Habitable Worlds is supported by the Pennsylvania State University, the Eberly College of Science, and the Pennsylvania Space Grant Consortium.

\section{REFERENCES}

[1] Koch, D. G., Borucki, W. J., Basri, G., Batalha, N. M., Brown, T. M., Caldwell, D., Christensen-Dalsgaard, J., Cochran, W. D., DeVore, E., Dunham, E. W., Gautier, Thomas N., I., Geary, J. C., Gilliland, R. L., Gould, A., Jenkins, J., Kondo, Y., Latham, D. W., Lissauer, J. J., Marcy, G., Monet, D., Sasselov, D., Boss, A., Brownlee, D., Caldwell, J., Dupree, A. K., Howell, S. B., Kjeldsen, H., Meibom, S., Morrison, D., Owen, T., Reitsema, H., Tarter, J., Bryson, S. T., Dotson, J. L., Gazis, P., Haas, M. R., Kolodziejczak, J., Rowe, J. F., Van Cleve, J. E., Allen, C., Chandrasekaran, H., Clarke, B. D., Li, J., Quintana, E. V., Tenenbaum, P., Twicken, J. D., and Wu, H., "Kepler Mission Design, Realized Photometric Performance, and Early Science," 713, L79-L86 (Apr. 2010). 
[2] Borucki, W. J., Koch, D., Basri, G., Batalha, N., Brown, T., Caldwell, D., Caldwell, J., ChristensenDalsgaard, J., Cochran, W. D., DeVore, E., Dunham, E. W., Dupree, A. K., Gautier, T. N., Geary, J. C., Gilliland, R., Gould, A., Howell, S. B., Jenkins, J. M., Kondo, Y., Latham, D. W., Marcy, G. W., Meibom, S., Kjeldsen, H., Lissauer, J. J., Monet, D. G., Morrison, D., Sasselov, D., Tarter, J., Boss, A., Brownlee, D., Owen, T., Buzasi, D., Charbonneau, D., Doyle, L., Fortney, J., Ford, E. B., Holman, M. J., Seager, S., Steffen, J. H., Welsh, W. F., Rowe, J., Anderson, H., Buchhave, L., Ciardi, D., Walkowicz, L., Sherry, W., Horch, E., Isaacson, H., Everett, M. E., Fischer, D., Torres, G., Johnson, J. A., Endl, M., MacQueen, P., Bryson, S. T., Dotson, J., Haas, M., Kolodziejczak, J., Van Cleve, J., Chandrasekaran, H., Twicken, J. D., Quintana, E. V., Clarke, B. D., Allen, C., Li, J., Wu, H., Tenenbaum, P., Verner, E., Bruhweiler, F., Barnes, J., and Prsa, A., "Kepler Planet-Detection Mission: Introduction and First Results," Science 327, 977 (Feb. 2010).

[3] Howell, S. B., Sobeck, C., Haas, M., Still, M., Barclay, T., Mullally, F., Troeltzsch, J., Aigrain, S., Bryson, S. T., Caldwell, D., Chaplin, W. J., Cochran, W. D., Huber, D., Marcy, G. W., Miglio, A., Najita, J. R., Smith, M., Twicken, J. D., and Fortney, J. J., "The K2 Mission: Characterization and Early Results," 126, 398 (Apr. 2014).

[4] Ricker, G. R., Winn, J. N., Vanderspek, R., Latham, D. W., Bakos, G. Á., Bean, J. L., Berta-Thompson, Z. K., Brown, T. M., Buchhave, L., Butler, N. R., Butler, R. P., Chaplin, W. J., Charbonneau, D., Christensen-Dalsgaard, J., Clampin, M., Deming, D., Doty, J., De Lee, N., Dressing, C., Dunham, E. W., Endl, M., Fressin, F., Ge, J., Henning, T., Holman, M. J., Howard, A. W., Ida, S., Jenkins, J. M., Jernigan, G., Johnson, J. A., Kaltenegger, L., Kawai, N., Kjeldsen, H., Laughlin, G., Levine, A. M., Lin, D., Lissauer, J. J., MacQueen, P., Marcy, G., McCullough, P. R., Morton, T. D., Narita, N., Paegert, M., Palle, E., Pepe, F., Pepper, J., Quirrenbach, A., Rinehart, S. A., Sasselov, D., Sato, B., Seager, S., Sozzetti, A., Stassun, K. G., Sullivan, P., Szentgyorgyi, A., Torres, G., Udry, S., and Villasenor, J., "Transiting Exoplanet Survey Satellite (TESS)," Journal of Astronomical Telescopes, Instruments, and Systems 1, 014003 (Jan. 2015).

[5] Bender, C. F., Akeson, R., Allen, L., Anderson, T. B., Bastien, F. A., Blake, C., Blakeslee, S., Chakraborty, A., Diddams, S. A., Gong, Q., Halverson, S., Hearty, F., Hunting, E., Jaehnig, K. P., Kanodia, S., Kaplan, K. F., Levi, E. I., Li, D., Luhn, J. K., Logsdon, S. E., Mahadevan, S., McElwain, M. W., Monson, A. J., P., N. J., Percival, J. W., Rajagopal, J., Ramsey, L. W., Roberston, P., Roy, A., Schwab, C., Smith, M. P., Stefánsson, G. K., Terrien, R. C., Wolf, M. J., and Wright, J. T., "The NEID precision radial velocity spectrometer: project overview and status update," Proc. SPIE Paper 10702-39, this conference (2018).

[6] Schwab, C., Rakich, A., Gong, Q., Mahadevan, S., Halverson, S. P., Roy, A., Terrien, R. C., Robertson, P. M., Hearty, F. R., Levi, E. I., Monson, A. J., Wright, J. T., McElwain, M. W., Bender, C. F., Blake, C. H., Stürmer, J., Gurevich, Y. V., Chakraborty, A., and Ramsey, L. W., "Design of NEID, an extreme precision Doppler spectrograph for WIYN," in [Proceedings of the SPIE, Volume 9908, id. $99087 \mathrm{H} 6 \mathrm{pp}$. (2016).], 9908 (Aug. 2016).

[7] Schwab, C., Liang, M., Gong, Q., Bender, C. F., Blake, C., Halverson, S. P., Harbeck, D. R., Hearty, F., Hunting, E., Jaehnig, K. P., Logsdon, S. E., Mahadevan, S., McElwain, M. W., Monson, A. J., Percival, J. W., Rajagopal, J., Ramsey, L. W., Roy, A., Santoro, F., Smith, M. P., Terrien, R. C., Timmerman, E., Wolf, M. J., and Wright, J. T., "The NEID precision radial velocity spectrometer: optical design of the port adapter and ADC," Proc. SPIE Paper 10702-257, this conference (2018).

[8] Percival, J. W., Gong, Q., Harbeck, D., Hunting, E., Jaehnig, K. P., Liang, M., Logsdon, S. E., McElwain, M. W., Rajagopal, J., Schwab, C., Smith, M. P., Timmerman, E., and Wolf, M. J., "The NEID precision radial velocity spectrometer: fast, first-order wavefront correction," Proc. SPIE Paper 10702-241, this conference (2018).

[9] G2 Engineering, "http://www.g2-engineering.com/."

[10] Halverson, S., Terrien, R., Mahadevan, S., Roy, A., Bender, C., Stefánsson, G. K., Monson, A., Levi, E., Hearty, F., Blake, C., McElwain, M., Schwab, C., Ramsey, L., Wright, J., Wang, S., Gong, Q., and Roberston, P., "A comprehensive radial velocity error budget for next generation Doppler spectrometers," in [Proceedings of the SPIE, Volume 9908, id. 99086 P 20 pp. (2016).], 9908 (Aug. 2016).

[11] Lightsey, P. A., Wei, Z., Skelton, D. L., Bowers, C. W., Mehalick, K. I., Thomson, S. R., Knollenberg, P., and Arenberg, J. W., "Stray light performance for the James Webb Space Telescope," in [Space Telescopes and Instrumentation 2014: Optical, Infrared, and Millimeter Wave], 9143, 91433P (Aug. 2014). 
[12] Pathak, P., Guyon, O., Jovanovic, N., Lozi, J., Martinache, F., Minowa, Y., Kudo, T., Takami, H., Hayano, Y., and Narita, N., "A High-precision Technique to Correct for Residual Atmospheric Dispersion in Highcontrast Imaging Systems," Publications of the Astronomical Society of the Pacific 128, 124404 (Dec. 2016).

[13] Mahadevan, S., Ramsey, L., Bender, C., Terrien, R., Wright, J. T., Halverson, S., Hearty, F., Nelson, M., Burton, A., Redman, S., Osterman, S., Diddams, S., Kasting, J., Endl, M., and Deshpande, R., "The habitable-zone planet finder: a stabilized fiber-fed NIR spectrograph for the Hobby-Eberly Telescope," in [Ground-based and Airborne Instrumentation for Astronomy IV], 8446, 84461S (Sept. 2012).

[14] Mahadevan, S., Ramsey, L. W., Terrien, R., Halverson, S., Roy, A., Hearty, F., Levi, E., Stefansson, G. K., Robertson, P., Bender, C., Schwab, C., and Nelson, M., "The Habitable-zone Planet Finder: A status update on the development of a stabilized fiber-fed near-infrared spectrograph for the for the Hobby-Eberly telescope," in [Ground-based and Airborne Instrumentation for Astronomy V], 9147, 91471G (July 2014). 